\title{
Hamiltonian Approach to the Modeling of Internal Geophysical Waves with Vorticity
}

\author{
Alan Compelli \\ Technological University Dublin, alan.compelli@tudublin.ie
}

Follow this and additional works at: https://arrow.tudublin.ie/scschmatart

Part of the Mathematics Commons, Non-linear Dynamics Commons, and the Partial Differential Equations Commons

\section{Recommended Citation}

Compelli, A. (2014). Hamiltonian Approach to the Modelling of Internal Geophysical Waves with Vorticity. Accepted for Publication in Monatshefte für Mathematik doi: 10.21427/29ra-c897

This Article is brought to you for free and open access by the School of Mathematics at ARROW@TU Dublin. It has been accepted for inclusion in Articles by an authorized administrator of ARROW@TU Dublin. For more information, please contact arrow.admin@tudublin.ie, aisling.coyne@tudublin.ie,gerard.connolly@tudublin.ie.

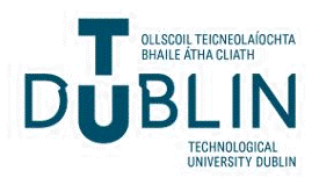




\title{
Hamiltonian Approach to the Modeling of Internal Geophysical Waves with Vorticity
}

\author{
Alan Compelli \\ School of Mathematical Sciences, \\ Dublin Institute of Technology, Kevin Street, \\ Dublin 8, \\ Ireland. \\ alan.compelli@mydit.ie
}

December 10, 2014

\begin{abstract}
We examine a simplified model of internal geophysical waves in a rotational 2-dimensional water-wave system, under the influence of Coriolis forces and with gravitationally induced waves. The system consists of a lower medium, bound underneath by an impermeable flat bed, and an upper lid. The 2 media have a free common interface. Both media have constant density and constant (non-zero) vorticity. By examining the governing equations of the system we calculate the Hamiltonian of the system in terms of its conjugate variables and perform a variable transformation to show that it has canonical Hamiltonian structure. We then linearize the system, determine the equations of motion of the linearized system and calculate the dispersion relation. Finally, limiting cases are examined to recover irrotational and single medium systems as well as an infinite 2 media system.

Keywords: Hamiltonian formulation; constant vorticity; geophysical waves; Coriolis forces; canonical structure; linearization

Mathematics Subject Classification (2000): 76B55; 76B15; 86A05; 76B47; 35Q31
\end{abstract}

\section{Introduction}

Internal geophysical waves are waves that propagate under the influence of gravity within a fluid body, e.g. within an ocean. Thermoclines are inter- 
faces between discrete fluid bodies characterized by significant temperature variations with change in depth. This phenomena is of particular interest in the studies of climatology, marine biology, etc. and is of significant importance to the fishing sector. This area has been reviewed by Fedorov and Brown [1].

The study of geophysical waves draws from several previous papers including the irrotational case, i.e. with zero vorticity, by Zakharov [2] which showed the canonical Hamiltonian structure of an infinitely deep fluid system with a free surface with gravitationally induced waves and also several subsequent papers including [3], [4], [5], [6], [7].

For studies of rotational systems, i.e. with non-zero constant vorticity, we refer to [8], [9], [10], [11], [12], [13], [14], [15].

However, the system under study is a 2 media system and so consideration is given to the work of Benjamin and Bridges [16], [17]. Craig et al. [18], [19] considered an irrotational system consisting of a lower medium bound underneath by a flat bed and an upper medium bound above by an impermeable lid such that the 2 media have a free common interface and also the case in which the upper medium itself has a free surface; see also [20] for the bounded rotational case.

Recent papers by Constantin [21], [22] have expanded existing knowledge by considering a rotational system with a free common interface and free surface under the influence of Earth's Coriolis force near the equator; see also [23], [24] for equatorial flows with continuous stratification.

The aim of this paper is to consider a bounded, 2 media, rotational system subject to gravity and Coriolis forces. The Hamiltonian of the system in terms of it's conjugate variables will be calculated, it will be examined for canonical Hamiltonian structure and it will be linearized.

\section{Preliminaries}

As per Figure 1 we define the lower medium $\Omega_{1}$ as the domain $\left\{(x, y) \in \mathbb{R}^{2}\right.$ : $\left.-h_{1}<y<\eta(x, t)\right\}$, the upper medium $\Omega_{2}$ as the domain $\left\{(x, y) \in \mathbb{R}^{2}\right.$ : $\left.\eta(x, t)<y<h_{2}\right\}$ and the entire system $\Omega_{1,2}$ as the domain $\left\{(x, y) \in \mathbb{R}^{2}\right.$ : $\left.-h_{1}<y<h_{2}\right\}$ where $\{y=\eta(x, t)\}$ describes the elevation of the common interface. The subscript $c$ will be used to denote evaluation at the common interface.

In physical reality the top surface will have surface waves, e.g. corresponding to an ocean surface. We can describe the elevation of these waves by $\{y=$ $\left.\eta_{1}(x, t)\right\}$. However, we assume that $(\eta)_{\max }>>\left(\eta_{1}\right)_{\max }$ and hence consider 


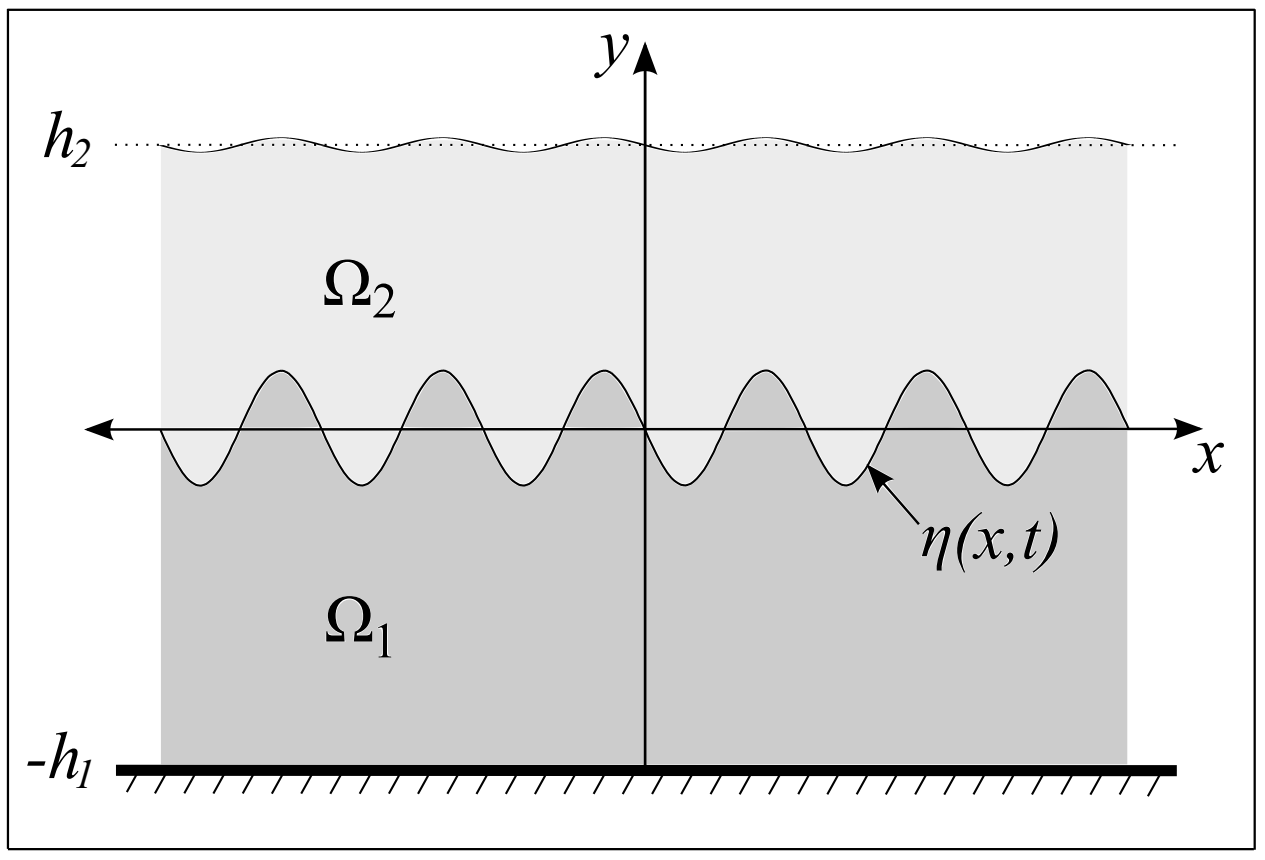

Figure 1: The system under study

the top surface to be a flat boundary like a rigid lid.

We use the subscript notation $i=\{1,2\}$ to represent the lower and upper media respectively and thus can consider a velocity potential $\varphi_{i}$ which is defined by:

$$
\left\{\begin{array}{l}
u_{i}=\partial_{x} \varphi_{i}-\gamma_{i} y \\
v_{i}=\partial_{y} \varphi_{i}
\end{array}\right.
$$

where non-lateral velocity flow, with propagation in the positive $x$-direction, is given by $\mathbf{V}_{\mathbf{i}}(x, y, z)=\left(u_{i}, v_{i}, 0\right)$ and $\gamma_{1}$ and $\gamma_{2}$ are the respective non-zero constant vorticities.

Additionally, the stream function $\psi_{i}$ is introduced, defined by:

$$
\left\{\begin{array}{l}
u_{i}=-\partial_{y} \psi_{i} \\
v_{i}=\partial_{x} \psi_{i} .
\end{array}\right.
$$

$\rho_{1}$ and $\rho_{2}$ are the respective constant densities of the lower and upper media and stability is given by the condition that

$$
\rho_{1}>\rho_{2} .
$$


We assume that for large $|x|$ the amplitude of $\eta$ attenuates and hence make the following assumptions

$$
\begin{gathered}
\lim _{|x| \rightarrow \infty} \eta(x, t)=0, \\
\lim _{|x| \rightarrow \infty} \varphi_{i}(x, y, t)=0,
\end{gathered}
$$

and

$$
-h_{1}<\eta(x, t)<h_{2} \text { for all } x \text { and } t .
$$

\section{Governing Equations}

We write Euler's equation as:

$$
\partial_{t} \mathbf{V}_{\mathbf{i}}+\left(\mathbf{V}_{\mathbf{i}} \cdot \nabla\right) \mathbf{V}_{\mathbf{i}}=-\frac{1}{\rho_{i}} \nabla P_{i}+\mathbf{g}+\mathbf{F}_{c}
$$

where $P_{i}=\rho_{i} g y+p_{\text {atm }}+p_{i}$ is the pressure at a depth $y, p_{\text {atm }}$ is (constant) atmospheric pressure, $p_{i}$ is the dynamic pressure due to the wave motion, $g$ is the acceleration due to gravity (where $y$ points in the opposite direction to the center of gravity), $\mathbf{g}$ is the force due to gravity per unit mass, and

$$
\mathbf{F}_{c}=-2 \omega \nabla \psi_{i}
$$

is the Coriolis force per unit mass, with $\omega$ the rotational speed of Earth. Applying Equations (1) and (2) this can be written as

$$
\nabla\left(\partial_{t} \varphi_{i}+\frac{1}{2}\left(\nabla \psi_{i}\right)^{2}-\gamma_{i} \psi_{i}+2 \omega \psi_{i}\right)=\nabla\left(-g y-\frac{p_{i}}{\rho_{i}}\right)
$$

where $\nabla=\left(\partial_{x}, \partial_{y}\right)$.

At the interface $p_{1}=p_{2}=p_{c}$ therefore we write Euler's equation in terms of the velocity potentials, stream functions, densities and vorticities as the energy conserving equality

$$
\begin{aligned}
\rho_{1} \nabla\left(\left(\partial_{t} \varphi_{1}\right)_{c}+\right. & \left.\frac{1}{2}\left(\nabla \psi_{1}\right)_{c}^{2}-\left(\gamma_{1}-2 \omega\right) \chi_{1}+g \eta\right) \\
& -\rho_{2} \nabla\left(\left(\partial_{t} \varphi_{2}\right)_{c}+\frac{1}{2}\left(\nabla \psi_{2}\right)_{c}^{2}-\left(\gamma_{2}-2 \omega\right) \chi_{2}+g \eta\right)=0
\end{aligned}
$$


where $\chi_{i}$ is the stream function evaluated at the interface.

The following Bernoulli condition at the interface follows from assumptions (4) and (5):

$$
\begin{aligned}
\rho_{1}\left(\left(\partial_{t} \varphi_{1}\right)_{c}+\frac{1}{2}\left(\nabla \psi_{1}\right)_{c}^{2}-\right. & \left.\left(\gamma_{1}-2 \omega\right) \chi_{1}+g \eta\right) \\
= & \rho_{2}\left(\left(\partial_{t} \varphi_{2}\right)_{c}+\frac{1}{2}\left(\nabla \psi_{2}\right)_{c}^{2}-\left(\gamma_{2}-2 \omega\right) \chi_{2}+g \eta\right) .
\end{aligned}
$$

We will also use the following kinematic boundary conditions

$$
\left\{\begin{array}{l}
\partial_{t} \eta-\partial_{x} \eta\left(\gamma_{i} \eta-\left(\partial_{x} \varphi_{i}\right)_{c}\right)-\left(\partial_{y} \varphi_{i}\right)_{c}=0 \\
\partial_{y}\left(\varphi_{1}\right)_{b}=\partial_{y}\left(\varphi_{2}\right)_{l}=0
\end{array}\right.
$$

noting that $\mathbf{V}_{\mathbf{1}}\left(x,-h_{1}, 0\right)=\left(u_{1}, 0,0\right)$ and $\mathbf{V}_{\mathbf{2}}\left(x, h_{2}, 0\right)=\left(u_{2}, 0,0\right)$, where the subscripts $b$ and $l$ denote evaluation at the bottom (lower boundary) and lid (upper boundary) respectively.

\section{Hamiltonian Formulation}

If we consider the system under study as an irrotational system the Hamiltonian, $H$, is given by the sum of the kinetic and potential energies as:

$$
H=\frac{1}{2} \int_{\mathbb{R}} \int_{-h_{1}}^{\eta} \rho_{1}\left(\nabla \varphi_{1}\right)^{2} d y d x+\frac{1}{2} \int_{\mathbb{R}} \int_{\eta}^{h_{2}} \rho_{2}\left(\nabla \varphi_{2}\right)^{2} d y d x+\frac{1}{2} \int_{\mathbb{R}}\left(\rho_{1}-\rho_{2}\right) g \eta^{2} d x .
$$

However, as we are concerned with the rotational case the Hamiltonian (noting the additional vorticity related terms) is given by:

$$
\begin{gathered}
H=\frac{1}{2} \int_{\mathbb{R}} \int_{-h_{1}}^{\eta} \rho_{1}\left(\nabla \varphi_{1}\right)^{2} d y d x+\frac{1}{2} \int_{\mathbb{R}} \int_{\eta}^{h_{2}} \rho_{2}\left(\nabla \varphi_{2}\right)^{2} d y d x+\frac{1}{2} \int_{\mathbb{R}}\left(\rho_{1}-\rho_{2}\right) g \eta^{2} d x \\
-\int_{\mathbb{R}} \int_{-h_{1}}^{\eta} \rho_{1} \gamma_{1} y \partial_{x} \varphi_{1} d y d x-\iint_{\mathbb{R}}^{h_{2}} \rho_{2} \gamma_{2} y \partial_{x} \varphi_{2} d y d x \\
+\frac{1}{2} \int_{\mathbb{R}} \int_{-h_{1}}^{\eta} \rho_{1} \gamma_{1}^{2} y^{2} d y d x+\frac{1}{2} \int_{\mathbb{R}} \int_{\eta}^{h_{2}} \rho_{2} \gamma_{2}^{2} y^{2} d y d x
\end{gathered}
$$


We introduce $\xi_{i}:=\left(\varphi_{i}\right)_{c}=\varphi_{i}(x, \eta(x, t), t)$ as the interface velocity potential and hence define [16], [17]

$$
\xi:=\rho_{1} \xi_{1}-\rho_{2} \xi_{2} .
$$

Also, by defining [18]

$$
B:=\rho_{1} G_{2}(\eta)+\rho_{2} G_{1}(\eta)
$$

and using the Dirichlet-Neumann operator $G_{i}(\eta)$ given by (see [4], [19])

$$
G_{i}(\eta) \xi_{i}=\partial_{\mathbf{n}_{i}} \varphi_{i} \sqrt{1+\left(\partial_{x} \eta\right)^{2}},
$$

where $\partial_{\mathbf{n}_{i}} \varphi_{i}$ is the normal derivative of the velocity potential $\varphi_{i}$, at the surface, for an outward normal $\mathbf{n}_{i}$, we can express the Hamiltonian in terms of the conjugate variables $(\eta, \xi)$ as (for details see [20]):

$$
\begin{gathered}
H(\eta, \xi)=\frac{1}{2} \int_{\mathbb{R}} \xi\left(G_{1}(\eta) B^{-1} G_{2}(\eta)\right) \xi d x+\frac{1}{2} \int_{\mathbb{R}}\left(\rho_{1}-\rho_{2}\right) g \eta^{2} d x \\
-\frac{1}{2} \int_{\mathbb{R}} \rho_{1} \rho_{2}\left(\gamma_{2}-\gamma_{1}\right)^{2} \eta \partial_{x} \eta B^{-1} \eta \partial_{x} \eta d x \\
+\int_{\mathbb{R}} \eta \partial_{x} \eta B^{-1}\left(\rho_{1} \gamma_{1} G_{2}(\eta) \xi+\rho_{2} \gamma_{2} G_{1}(\eta) \xi\right) d x+\frac{1}{6} \int_{\mathbb{R}}\left(\rho_{1} \gamma_{1}^{2}-\rho_{2} \gamma_{2}^{2}\right) \eta^{3} d x .
\end{gathered}
$$

Using the Hamiltonian from (14) its variation is given by

$$
\begin{gathered}
\delta H=\int_{\mathbb{R}} \int_{-h_{1}}^{\eta} \rho_{1}\left(\nabla \varphi_{1}\right) \cdot \nabla \delta \varphi_{1} d y d x+\int_{\mathbb{R}} \int_{\eta}^{h_{2}} \rho_{2}\left(\nabla \varphi_{2}\right) \cdot \nabla \delta \varphi_{2} d y d x \\
+\frac{1}{2} \int_{\mathbb{R}} \rho_{1}\left(\nabla \varphi_{1}\right)_{c}^{2} \delta \eta d x-\frac{1}{2} \int_{\mathbb{R}} \rho_{2}\left(\nabla \varphi_{2}\right)_{c}^{2} \delta \eta d x+\int_{\mathbb{R}}\left(\rho_{1}-\rho_{2}\right) g \eta \delta \eta d x \\
-\int_{\mathbb{R}} \int_{-h_{1}}^{\eta} \rho_{1} \gamma_{1} y \delta\left(\partial_{x} \varphi_{1}\right) d y d x-\int_{\mathbb{R}} \int_{\eta}^{h_{2}} \rho_{2} \gamma_{2} y \delta\left(\partial_{x} \varphi_{2}\right) d y d x \\
-\int_{\mathbb{R}}\left(\rho_{1} \gamma_{1} \eta\left(\partial_{x} \varphi_{1}\right)_{c}-\rho_{2} \gamma_{2} \eta\left(\partial_{x} \varphi_{2}\right)_{c}\right) \delta \eta d x+\frac{1}{2} \int_{\mathbb{R}}\left(\rho_{1} \gamma_{1}^{2} \eta^{2}-\rho_{2} \gamma_{2}^{2} \eta^{2}\right) \delta \eta d x .
\end{gathered}
$$


From (12), at the interface, $\partial_{t} \eta=\partial_{x}\left(\psi_{i}(x, \eta, t)\right)$ and so we can introduce

$$
\chi:=\chi_{1}=\chi_{2}
$$

and also the new variable

$$
\Gamma:=\rho_{1} \gamma_{1}-\rho_{2} \gamma_{2}-2 \omega\left(\rho_{1}-\rho_{2}\right)
$$

which, by also applying the Bernoulli condition (11), gives the non-canonical system

$$
\left\{\begin{array}{l}
\partial_{t} \eta=\delta_{\xi} H \\
\partial_{t} \xi=-\delta_{\eta} H+\Gamma \chi
\end{array}\right.
$$

Using [15]

$$
\chi(x, t)=\int_{-\infty}^{x} \frac{\delta H}{\delta \xi\left(x^{\prime}\right)} d x^{\prime}
$$

and

$$
\int_{\mathbb{R}} \eta(x, t) d x=\text { constant }
$$

we can write (22) as:

$$
\left\{\begin{array}{l}
\partial_{t} \eta=\delta_{\xi} H \\
\partial_{t} \xi=-\delta_{\eta} H+\Gamma \int_{-\infty}^{x} \eta_{t} d x^{\prime}
\end{array}\right.
$$

From [15] it is known that this system of equations has a Hamiltonian form

$$
\left\{\begin{array}{l}
\partial_{t} \eta=\{\eta, H\} \\
\partial_{t} \xi=\{\xi, H\}
\end{array}\right.
$$

where the Poisson bracket in [15] is defined as

$$
\{A, B\}=\int_{\mathbb{R}}\left(\frac{\delta A}{\delta \eta(x)} \frac{\delta B}{\delta \xi(x)}-\frac{\delta A}{\delta \xi(x)} \frac{\delta B}{\delta \eta(x)}\right) d x+\Gamma \int_{\mathbb{R}}\left(\frac{\delta A}{\delta \xi(x)} \int_{0}^{x} \frac{\delta B}{\delta \xi\left(x^{\prime}\right)} d x^{\prime}\right) d x .
$$

One can verify that (27) satisfies all properties of a Poisson bracket. However, we will establish this by a variable transformation. The velocity potential 
at the interface, $\xi$, is defined modulo an additive constant. We chose to transform it, as per the single media case [15], to a new variable $\zeta$ as follows

$$
\xi \rightarrow \zeta=\xi-\frac{\Gamma}{2} \int_{-\infty}^{x} \eta\left(x^{\prime}, t\right) d x^{\prime} .
$$

The system under study has canonical Hamiltonian structure. In general,

$$
\{A, B\}=\int_{\mathbb{R}}\left(\frac{\delta A}{\delta \eta(x)} \frac{\delta B}{\delta \zeta(x)}-\frac{\delta A}{\delta \zeta(x)} \frac{\delta B}{\delta \eta(x)}\right) d x .
$$

This indeed shows that (27) defines a (non-canonical) Poisson bracket in terms of the variables $(\eta, \xi)$.

\section{Linearization of the Hamiltonian}

By Taylor expanding the Dirichlet-Neumann operator we can represent it in terms of orders of $\eta$ as

$$
G_{i}(\eta)=\sum_{j=0}^{\infty} G_{i j}(\eta)
$$

with the constant, linear and quadratic terms given as [18]:

$$
\begin{aligned}
G_{i 0} & =D \tanh \left(h_{i} D\right) \\
G_{11}(\eta) & =D \eta D-G_{10} \eta G_{10} \\
G_{21}(\eta) & =-D \eta D+G_{20} \eta G_{20} \\
G_{i 2}(\eta) & =-\frac{1}{2}\left(D^{2} \eta^{2} G_{i 0}-2 G_{i 0} \eta G_{i 0} \eta G_{i 0}+G_{i 0} \eta^{2} D^{2}\right)
\end{aligned}
$$

where the operator $D$ is a Fourier multiplier equivalent to both the operation $-i \partial_{x}$ and the wavenumber $k$, i.e.

$$
D=-i \partial_{x}=k .
$$

The operator $B$ can therefore be expressed as

$$
B=\rho_{1} \sum_{j=0}^{\infty} G_{2 j}(\eta)+\rho_{2} \sum_{j=0}^{\infty} G_{1 j}(\eta),
$$


and, also, the Hamiltonian can be represented as

$$
H(\eta, \xi)=\sum_{j=0}^{\infty} \mu^{j} H^{(j)}\left(\eta^{*}, \xi^{*}\right) .
$$

Using equations (31)-(37) the Hamiltonian (18) can hence be expressed as

$$
\begin{gathered}
H(\eta, \xi)=\frac{1}{2} \int_{\mathbb{R}} \xi\left(G_{10}+\mathcal{O}(\eta)\right)\left(\rho_{1} G_{20}+\rho_{2} G_{10}+\mathcal{O}(\eta)\right)^{-1}\left(G_{20}+\mathcal{O}(\eta)\right) \xi d x \\
-\frac{1}{2} \int_{\mathbb{R}} \rho_{1} \rho_{2}\left(\gamma_{2}-\gamma_{1}\right)^{2} \eta \partial_{x} \eta\left(\rho_{1} G_{20}+\rho_{2} G_{10}+\mathcal{O}(\eta)\right)^{-1} \eta \partial_{x} \eta d x \\
+\int_{\mathbb{R}} \eta \partial_{x} \eta\left(\rho_{1} G_{20}+\rho_{2} G_{10}+\mathcal{O}(\eta)\right)^{-1}\left(\rho_{1} \gamma_{1}\left(G_{20}+\mathcal{O}(\eta)\right) \xi+\rho_{2} \gamma_{2}\left(G_{10}+\mathcal{O}(\eta)\right) \xi\right) d x \\
+\frac{1}{2} \int_{\mathbb{R}}\left(\rho_{1}-\rho_{2}\right) g \eta^{2} d x+\frac{1}{6} \int_{\mathbb{R}}\left(\rho_{1} \gamma_{1}^{2}-\rho_{2} \gamma_{2}^{2}\right) \eta^{3} d x .
\end{gathered}
$$

Next, we define transformed parameters in terms of a small arbitrary constant parameter $\mu$ such that

$$
\left\{\begin{array}{l}
\eta^{*}=\mu^{-1} \eta \\
\xi^{*}=\mu^{-1} \xi \\
x^{*}=\mu^{-1} x
\end{array}\right.
$$

and hence $B^{*}$, the transformation of $B$, is given as:

$$
B^{*}=\rho_{1}\left(G_{20}+\mathcal{O}\left(\eta^{*}\right)\right)+\rho_{2}\left(G_{10}+\mathcal{O}\left(\eta^{*}\right)\right),
$$


and we can write the Hamiltonian as:

$$
\begin{gathered}
H\left(\eta^{*}, \xi^{*}\right) \\
=\frac{1}{2} \int_{\mathbb{R}} \mu \xi^{*}\left(G_{10}+\mathcal{O}\left(\eta^{*}\right)\right)\left(\rho_{1} G_{20}+\rho_{2} G_{10}+\mathcal{O}\left(\eta^{*}\right)\right)^{-1}\left(G_{20}+\mathcal{O}\left(\eta^{*}\right)\right) \mu \xi^{*} d x \\
-\frac{1}{2} \int_{\mathbb{R}} \rho_{1} \rho_{2}\left(\gamma_{2}-\gamma_{1}\right)^{2} \mu \eta^{*} \mu \partial_{x} \eta^{*}\left(\rho_{1} G_{20}+\rho_{2} G_{10}+\mathcal{O}\left(\eta^{*}\right)\right)^{-1} \mu \eta^{*} \mu \partial_{x} \eta^{*} d x \\
\quad+\int_{\mathbb{R}} \mu \eta^{*} \mu \partial_{x} \eta^{*}\left(\rho_{1} G_{20}+\rho_{2} G_{10}+\mathcal{O}\left(\eta^{*}\right)\right)^{-1} \\
\left(\rho_{1} \gamma_{1}\left(G_{20}+\mathcal{O}\left(\eta^{*}\right)\right) \mu \xi^{*}+\rho_{2} \gamma_{2}\left(G_{10}+\mathcal{O}\left(\eta^{*}\right)\right) \mu \xi^{*}\right) d x \\
+\frac{1}{2} \int_{\mathbb{R}}\left(\rho_{1}-\rho_{2}\right) g \mu^{2} \eta^{* 2} d x+\frac{1}{6} \int_{\mathbb{R}}\left(\rho_{1} \gamma_{1}^{2}-\rho_{2} \gamma_{2}^{2}\right) \mu^{3} \eta^{* 3} d x .
\end{gathered}
$$

Separating all terms of $\mathcal{O}\left(\mu^{3}\right)$ and recalling the definition of $G_{i 0}$ from (31) we can write this as:

$$
\begin{aligned}
H\left(\eta^{*}, \xi^{*}\right)=\frac{1}{2} \mu^{2} \int_{\mathbb{R}} \xi^{*} \frac{D \tanh \left(h_{1} D\right) \tanh \left(h_{2} D\right)}{\rho_{1} \tanh \left(h_{2} D\right)+\rho_{2} \tanh \left(h_{1} D\right)} \xi^{*} d x \\
\quad+\frac{1}{2} \mu^{2} \int_{\mathbb{R}}\left(\rho_{1}-\rho_{2}\right) g \eta^{* 2} d x+\mathcal{O}\left(\mu^{3}\right) .
\end{aligned}
$$

Therefore $H^{(2)}$ in terms of $\eta$ and $\xi$ is given by

$$
\begin{aligned}
H^{(2)}(\eta, \xi)=\frac{1}{2} \int_{\mathbb{R}} \xi \frac{D \tanh \left(h_{1} D\right) \tanh \left(h_{2} D\right)}{\rho_{1} \tanh \left(h_{2} D\right)+\rho_{2} \tanh \left(h_{1} D\right)} \xi d x & \\
& +\frac{1}{2} \int_{\mathbb{R}}\left(\rho_{1}-\rho_{2}\right) g \eta^{2} d x .
\end{aligned}
$$

Recalling (25) we calculate the linearized equations of motion as

$$
\begin{aligned}
& \partial_{t} \eta=\frac{D \tanh \left(h_{1} D\right) \tanh \left(h_{2} D\right)}{\rho_{1} \tanh \left(h_{2} D\right)+\rho_{2} \tanh \left(h_{1} D\right)} \xi \\
& \partial_{t} \xi=-\left(\rho_{1}-\rho_{2}\right) g \eta+\Gamma \int_{-\infty}^{x} \eta_{t} d x^{\prime} .
\end{aligned}
$$




\section{Dispersion relation of the linearized system}

Remark. The case with periodic boundary conditions can be treated similarly to the problem with decaying to zero conditions at $\pm \infty$. The same results remain valid when all quantities take values in the class of periodic functions of period $L$. Then one can consider only a domain with $0<x<L$ and replace $\int_{\mathbb{R}} d x$ with $\int_{0}^{L} d x$. (28) changes into $\zeta=\xi-\frac{\gamma}{2} \int_{0}^{x}\left(\eta\left(x^{\prime}, t\right)-\bar{\eta}\right) d x^{\prime}$ where $\bar{\eta}=\frac{1}{L} \int_{0}^{L} \eta(x, t) d x$ is the average of $\eta$. This is in order to preserve the periodicity, since the potential $\xi$ is determined up to a constant. The details are given in [15].

As $\eta$ and $\xi$ can be considered as $x$-periodic functions they can be represented as

$$
\left\{\begin{array}{l}
\eta(x, t)=\eta_{0} e^{i(k x-\Omega(k) t)} \\
\xi(x, t)=\xi_{0} e^{i(k x-\Omega(k) t)}
\end{array}\right.
$$

where $k$ is the wavenumber and $\Omega(k)$ is the angular frequency. Therefore

$$
\left\{\begin{array}{l}
\partial_{t} \eta=-i \Omega \eta \\
\partial_{t} \xi=-i \Omega \xi
\end{array}\right.
$$

and

$$
\int_{-\infty}^{x} \partial_{t} \eta d x^{\prime}=\frac{-i \Omega}{i k} \eta=-c \eta
$$

where

$$
c(k)=\frac{\Omega(k)}{k}
$$

is the wavespeed. Hence, by comparison with (44) and (45), we can write, noting that from (35) we are using $k$ instead of $D$,

$$
\left\{\begin{aligned}
-i \Omega \eta & =\frac{k \tanh \left(h_{1} k\right) \tanh \left(h_{2} k\right)}{\rho_{1} \tanh \left(h_{2} k\right)+\rho_{2} \tanh \left(h_{1} k\right)} \xi \\
-i \Omega \xi & =-\left(\rho_{1}-\rho_{2}\right) g \eta-\Gamma c \eta
\end{aligned}\right.
$$

By a straightforward calculation we can eliminate $\eta$ and $\xi$ and write the wavespeed as

$$
c=\frac{\tanh \left(h_{1} k\right) \tanh \left(h_{2} k\right)}{\rho_{1} \tanh \left(h_{2} k\right)+\rho_{2} \tanh \left(h_{1} k\right)}\left(\frac{\Gamma}{k}+\frac{g}{c k}\left(\rho_{1}-\rho_{2}\right)\right) .
$$


Multiplying across by $c$ gives us the quadratic expression

$$
\begin{aligned}
& c^{2}-c \frac{\tanh \left(h_{1} k\right) \tanh \left(h_{2} k\right)}{\rho_{1} \tanh \left(h_{2} k\right)+\rho_{2} \tanh \left(h_{1} k\right)}\left(\frac{\Gamma}{k}\right) \\
& -\frac{\tanh \left(h_{1} k\right) \tanh \left(h_{2} k\right)}{\rho_{1} \tanh \left(h_{2} k\right)+\rho_{2} \tanh \left(h_{1} k\right)}\left(\frac{g}{k}\left(\rho_{1}-\rho_{2}\right)\right)=0,
\end{aligned}
$$

with solutions

$$
c(k, \Gamma)=f_{1}(k, \Gamma) \pm \sqrt{f_{1}^{2}(k, \Gamma)+f_{2}(k)}
$$

where

$$
f_{1}(k, \Gamma)=\frac{1}{2} \frac{\tanh \left(h_{1} k\right) \tanh \left(h_{2} k\right)}{\rho_{1} \tanh \left(h_{2} k\right)+\rho_{2} \tanh \left(h_{1} k\right)}\left(\frac{\Gamma}{k}\right)
$$

and

$$
f_{2}(k)=\frac{\tanh \left(h_{1} k\right) \tanh \left(h_{2} k\right)}{\rho_{1} \tanh \left(h_{2} k\right)+\rho_{2} \tanh \left(h_{1} k\right)}\left(\frac{g}{k}\left(\rho_{1}-\rho_{2}\right)\right) .
$$

From assumption (3) $\rho_{1}>\rho_{2}$ and hence we have the solutions

$$
\left\{\begin{array}{l}
c_{+}(k, \Gamma)>0 \\
c_{-}(k, \Gamma)<0
\end{array}\right.
$$

which correspond to right and left moving waves respectively.

We consider the following limiting cases. Firstly, zero vorticity and zero Coriolis force, i.e. $\Gamma=0$,

$$
\Rightarrow c^{2}=\frac{\tanh \left(h_{1} k\right) \tanh \left(h_{2} k\right)}{\rho_{1} \tanh \left(h_{2} k\right)+\rho_{2} \tanh \left(h_{1} k\right)}\left(\frac{g}{k}\left(\rho_{1}-\rho_{2}\right)\right) .
$$

Secondly, zero vorticity, zero Coriolis force and infinite media. $\Gamma=0$ and as $h_{i} \rightarrow \infty$ then $\tanh \left(h_{i}\right) \rightarrow 1$ (cf. [16], [17])

$$
\Rightarrow c^{2}=\frac{g}{k} \frac{\left(\rho_{1}-\rho_{2}\right)}{\left(\rho_{1}+\rho_{2}\right)} .
$$

Lastly, zero vorticity, zero Coriolis force and consideration of the system as a single media system, i.e. $\rho_{2} \rightarrow 0$

$$
\Rightarrow c^{2}=\tanh \left(h_{1} k\right) \frac{g}{k},
$$

which is the well known dispersion relation for the linear approximation of gravity water waves in a single medium irrotational system. 


\section{Conclusion}

A closed water-wave system provided a simplified model for internal geophysical waves. The system that was considered consisted of 2 immiscible media with constant (non-zero) vorticities and constant densities separated by a common free interface. The lower medium was bounded below by an impermeable boundary and the upper medium, analogous to the surface of the sea, was assumed to have negligible surface wave elevation such that it was essentially a rigid lid. By examining non-lateral 2-dimensional flow, with gravitationally induced waves, and by considering the effects of the Coriolis force, the system governing equations were used to derive the Hamiltonian form of the system and the equations of motion in terms of phase space variables $(\eta, \xi)$ with non-canonical Hamiltonian structure. This was achieved by defining an interface velocity potential, $\xi$, in terms of the respective interface velocity potentials and constant densities of the 2 domains.

Moreover, by performing a variable transformation it was then shown that the system actually has canonical Hamiltonian structure with canonical phase space variables $(\eta, \zeta)$.

The system was then linearized and the equations of motion of the linearized system were calculated. The system was then considered as a dispersive medium and the dispersion relation was calculated. Finally the dispersion relation was considered under limiting behaviors to produce more common dispersion relations.

Further to the obtained results, non-linear effects and non-linear model equations need to be studied, like in [15], [18], [19], [25], etc. The problem of instability of the waves, as recently pursued in the publications [26], [27], [28] would be of interest.

\section{Acknowledgements}

The author would like to thank R. Ivanov and E. Prodanov at the Dublin Institute of Technology for their invaluable discussions on matters relating to this article and also thank an anonymous referee for important suggestions which have contributed to improvements in the overall quality of the article.

\section{References}

[1] A. Fedorov, J. Brown, Equatorial waves, Encyclopedia of Ocean Sciences (2009) 3679-3695. 
[2] V. Zakharov, Stability of periodic waves of finite amplitude on the surface of a deep fluid, Zh. Prikl. Mekh. Tekh. Fiz. 9 (1968) 86-94.

[3] T. Benjamin, P. Olver, Hamiltonian structure, symmetries and conservation laws for water waves, J. Fluid Mech. 125 (1982) 137-185.

[4] W. Craig, Water waves, Hamiltonian systems and Cauchy integrals, IMA Vol. Math. Appl. 30 (1991) 37-45.

[5] D. Milder, A note regarding 'On Hamilton's principle for water waves', J. Fluid Mech. 83 (1977) 159-161.

[6] J. Miles, Hamiltonian formulations for surface waves, Appl. Sci. Res. 37 (1981) 103-110.

[7] J. Miles, On Hamilton's principle for water waves, J. Fluid Mech. 83 (1977) 153-158.

[8] A. Constantin, On the deep water wave motion, J. Phys. A 34 (2001) $1405-1417$.

[9] A. Constantin, J. Escher, Symmetry of steady periodic surface water waves with vorticity, J. Fluid Mech. 498 (2004) 171-181.

[10] A. Constantin, D. Sattinger, W. Strauss, Variational formulations for steady water waves with vorticity, J. Fluid Mech. 548 (2006) 151-163.

[11] A. Constantin, W. Strauss, Exact steady periodic water waves with vorticity, Comm.Pure Appl. Math. 57 (2004) 481-527.

[12] A. Constantin, J. Escher, Analyticity of periodic traveling free surface water waves with vorticity, Ann. of Math. 173 (2011) 559-568.

[13] A. Teles da Silva, D. Peregrine, Steep, steady surface waves on water of finite depth with constant vorticity, J. Fluid Mech. 195 (1988) 281-302.

[14] A. Constantin, R. Ivanov, E. Prodanov, Nearly-Hamiltonian structure for water waves with constant vorticity, J. Math. Fluid Mech. 9 (2007) $1-14$.

[15] E. Wahlén, A Hamiltonian formulation of water waves with constant vorticity, Lett. Math. Phys. 79 (2007) 303-315.

[16] T. Benjamin, T. Bridges, Reappraisal of the Kelvin-Helmholtz problem. Part 1. Hamiltonian structure, J. Fluid Mech. 333 (1997) 301-325. 
[17] T. Benjamin, T. Bridges, Reappraisal of the Kelvin-Helmholtz problem. Part 2. Interaction of the Kelvin-Helmholtz, superharmonic and Benjamin-Feir instabilities, J. Fluid Mech. 333 (1997) 327-373.

[18] W. Craig, P. Guyenne, H. Kalisch, Hamiltonian long wave expansions for free surfaces and interfaces, Comm. Pure Appl. Math. 24 (2005) $1587-1641$.

[19] W. Craig, P. Guyenne, C. Sulem, Coupling between internal and surface waves, Nat. Hazards 57 (2011) 617-642.

[20] A. Compelli, Hamiltonian formulation of 2 bounded immiscible media with constant non-zero vorticities and a common interface, Wave Motion (2015) To be published.

[21] A. Constantin, On the modelling of equatorial waves, Geophys. Res. Lett. 39 (2012) L05602.

[22] A. Constantin, Some nonlinear, equatorially trapped, non-hydrostatic, internal geophysical waves, J. Phys. Oceanogr. 44 (2014) 781-789.

[23] A. Constantin, An exact solution for equatorially trapped waves, J. Geophys. Res. Oceans 117 (2012) C05029.

[24] D. Henry, An exact solution for equatorial geophysical water waves with an underlying current, Eur. J. Mech. B Fluids 38 (2013) 18-21.

[25] D. Henry, R. Ivanov, One-dimensional weakly nonlinear model equations for Rossby waves, Discrete Contin. Dyn. Syst. A 34 (2014) 3025-3034.

[26] D. Henry, H.-C. Hsu, Instability of equatorial water waves in the f-plane, Discrete Contin. Dyn. Syst. 35 (2015) 906-916.

[27] A. Constantin, P. Germain, Instability of some equatorially trapped waves, J. Geophys. Res. Oceans 118 (2013) 2802-2810.

[28] F. Genoud, D. Henry, Instability of equatorial water waves with an underlying current, J. Math. Fluid Mech. 16 (2014) 661-667. 